\title{
Beyond Ibrutinib Resistance in Chronic Lymphocytic Leukemia
}

\author{
Nahla A M Hamed* \\ Faculty of Medicine, Alexandria University, Egypt \\ Submission: April 09, 2017; Published: April 13, 2017 \\ "Correspondence Address: Nahla A M Hamed, Faculty of Medicine, Alexandria University, Egypt
}

\section{Abstract}

Despite ibrutinib activity in multiple B-cell malignancies, cases of primary and secondary resistance have emerged. The overall reported frequency of resistance is low. Resistance has been found to be caused by a mutation in the BTK binding site of ibrutinib or gain-of-function mutations in PLCG2, which lead to autonomous BCR activity. Improved understanding of mechanisms of primary and secondary resistance is essential. There are emerging alternative mechanisms that bypass BTK entirely and offer new opportunities for other targeted agents.

Abbreviations: BTK: Bruton's Tyrosine Kinase; FDA: Food and Drug Administration; MCL: Mantle Cell Lymphoma; CLL: Chronic Lymphocytic Leukemia; DLBCL: Diffuse Large B Cell Lymphoma; ABC: Activated B Cell; BCR: B-Cell Receptor; PLCG2: Phospholipase-CG2; SYK: Spleen Tyrosine Kinase; PI3K: Phosphatidyl Inositol-3 Kinase.

\section{Introduction}

BTKisa cytoplasmicenzymethatislocated inallhematopoietic cells (except for plasma cells and T-cell lymphocytes) [1]. BTK is a member of the Tec family kinases, which is a core component for B-cell differentiation and proliferation via downstream signalling of the BCR. Furthermore, BTK is also involved with signalling via CXCR4 and CXCR5 chemokine receptors, integrins and adhesion molecules that are essential for B-cell homing and trafficking [2].

Ibrutinib is a novel, first-in-class oral highly specific BTK inhibitor [3] that covalently binds to a cysteine residue at position 481 on BTK active site, [2] leading to an irreversible inhibition at Tyr-223 [1] and abrogates downstream signalling through BTK [2]. BTK inhibition by ibrutinib prevents malignant B-cell substrate adhesion, migration, proliferation, and survival [1].

\section{Ibrutinib and B-cell malignancies}

Clinical trials of ibrutinib have shown good activity in patients with relapsed MCL, with phase III data showing better tolerability and improvements in progression-free survival (14.6 months) [3]. Ibrutinib is approved by the United States FDA on November 2013 for relapsed and refractory MCL [3]. It has also been approved for treatment of Waldenstrom's macroglobulinemia [3] and CLL that harbours mutations in TP53 or deletion of $17 p$ for relapsed or refractory disease and also for first-line treatment [4]. In addition, ibrutinib has shown efficacy in subsets of patients with DLBCL (ABC-like subtype) [5].

\section{Ibrutinib and CLL}

In CLL, BTK is aberrantly activated, leading to over activation of cell survival pathways such as NF-kB and MAPK [2]. Ibrutinib induces CLL cells apoptosis directly via inhibition of BCR signalling and indirectly by inhibiting survival stimuli, fibronectin engagement, and stromal contact, thus preventing a niche and homing signal for CLL cells [2].

Ibrutinib characteristically causes rapid resolution of enlarged lymph nodes in CLL and an early redistribution of tissue-resident CLL cells into the peripheral blood, with a lymphocytosis surge. This lymphocytosis is asymptomatic, and resolves in most patients during the first few months of therapy [6]. Limited information is available on the bone marrow effects of ibrutinib. A gradual improvement of cytopenias provides indirect evidence that ibrutinib induces tumor regression in the bone marrow compartment. The response rate in the bone marrow was $62 \%$ after a median treatment time of 9.5 months; more and deeper remissions are likely to occur over time during prolonged therapy [7].

Although the clinical data demonstrate extremely encouraging responses, the majority of ibrutinib-treated patients do not achieve complete remission, and the lymphocyte counts stabilize in the long term in many patients at levels that are significantly lower than before treatment, but higher than normal [6]. Ibrutinib has shown clinically significant activity in relapsed CLL, with $71 \%$ of patients having an objective complete 
or partial response and an additional 15 to $20 \%$ of patients having a partial response with persistent lymphocytosis. At 26 months, the estimated progression-free survival rate among patients treated with ibrutinib is 75\% [8]. Resistance to ibrutinib, occurs particularly in heavily pretreated patients and in highrisk CLL [4]. It is important to differentiate ibrutinib resistance from ibrutinib intolerance, as patients who discontinue ibrutinib therapy because of side effects have a very different natural history [9].

\section{Adverse effects that warrant consideration prior to initiation of therapy}

Ibrutinib was very well tolerated [7]. Toxicities have been minimal and generally do not include myelosuppression due to the restricted expression of BTK to B cells [5]. The majority of adverse effects were grade 1 or 2 in nature, with diarrhea (47\%), upper respiratory tract infection (33\%), and fatigue (28\%) being the most common [2]. Ibrutinib has an incidence of atrial fibrillation of 3.5-6.5\% in CLL and also in Waldenstrom's macroglobulinemia and mantle cell lymphoma in clinical trials. It is hypothesized that ibrutinib induced atrial fibrillation due to the presence of BTK and related kinases on atrial tissue. Furthermore, PI3K-AKT signalling, a downstream pathway of BTK, has been found to be protective in the setting of cardiac stress [2] and is also thought to play an important role in the prevention of stress-induced cardiomyopathy [10].

In addition, patients exhibited increased bleeding events [2] most commonly petechiae and ecchymosis [11], despite improvement in their platelet counts as their CLL responded to therapy. Ibrutinib did not demonstrate any effects on prothrombin time or other key coagulation parameters. BTK is a mediator of platelet glycoprotein VI signalling and is irreversibly inhibited by ibrutinib. Platelets exhibited reduced function in response to collagen exposure and the degree of platelet inhibition was correlated with clinical bleeding. It is recommended to hold ibrutinib for 3 to 7 days and avoid concomitant warfarin prior to planned procedures [2]. Nonetheless, given the short half-life of ibrutinib, administering platelet transfusion approximately 6 $\mathrm{h}$ following a dose should reverse the hemostatic defect in the event of an emergency situation [2].

Potentially fatal cases of renal failure have also been associated with ibrutinib. Renal function should be periodically monitored and patients should be encouraged to stay adequately hydrated. Second primary malignancies, such as skin cancer and carcinomas have also occurred in up to $10 \%$ of patients on ibrutinib [2].

\section{Ibrutinib resistance}

Despite ibrutinib activity in multiple B-cell malignancies, the reported frequency of overall resistance is relatively low, but follow-up in many trials was short. Most patients now receive ibrutinib therapy outside of clinical trials and the exact incidence remains to be determined [5].
In primary resistance, patients demonstrate lack of response at initial therapy, whereas secondary resistance is characterized by loss of an initial disease response [5]. Unfortunately, this resistance correlates with aggressive disease, either progressive CLL or Richter transformation, that is difficult to treat. It is therefore of prime importance to detect the emergence of resistant disease early or even pre-emptively, because the affected patients will require biologically tailored alternative treatment strategies [4].

Relapse on ibrutinib occurs in two forms: progressive CLL and histologic transformation, most commonly to large cell lymphoma or prolymphocytic leukemia. Transformation generally occurs within the first 2 years of therapy, whereas CLL progression almost never occurs during the first year of treatment and the incidence continues to increase with time. Baseline karyotypic complexity on stimulated karyotype appears to be the most significant independent predictor for ibrutinib relapse with CLL [9].

BTK and PLCG2 mutations are not common at transformation. In contrast, patients with CLL progression are frequently associated with mutations of BTK and PLCG2. There may be a third group of patients with CLL progression without BTK or PLCG2 mutations [7]. Resistance to ibrutinib often involves mutation of a cysteine residue where ibrutinib binding occurs (8) resulting in irreversible inhibition of its kinase activity [12]. This finding, combined with two additional mutations in PLCG2 that are immediately downstream of BTK, underscores the importance of BCR pathway in the mechanism of action of ibrutinib in CLL [8]. Mutations in BTK and/or PLCG2 are present in $85-90 \%$ of patients at relapse when using high sensitivity assays. BTK C481S, the most common acquired mutation in BTK, reduces the binding affinity of ibrutinib for BTK and changes ibrutinib from an irreversible to a reversible inhibitor. The mutations identified in PLCG2 have all been demonstrated to be potentially gain-of-function, allowing activation through the BCR even in the presence of inactive BTK. Clonal evolution has also been shown to be a hallmark of ibrutinib resistance, and a recurrent deletion in $8 p$ in patients at the time of relapse have been noted. Current data suggest that patients with acquired resistance to ibrutinib have a poor survival [9].

An unresolved issue is the oftentimes low allelic fraction of mutations of BTK and PLCG2 that has been observed in patients with resistant CLL. Concurrent mutation of BTK and PLCG2 suggests either that the mutations are present in different subclones or that an additional activation of PLCG2 is advantageous for bypassing BTK inhibition because mutation of PLCG2 does not occur first in all patients [4].

\section{Treatment of CLL patients after ibrutinib relapse}

The most promising data for ibrutinib resistant patients are with the BCL2 inhibitor venetoclax. Overall response rate among ibrutinib refractory patients was $70 \%$ in a study of single agent 
venetoclax. The estimated PFS at 12 month was $80 \%$ for patients previously treated with ibrutinib. A "real world" experience of patients treated with idelalisib upon ibrutinib resistance showed an ORR of $28 \%$ with a median PFS of 8 months [9]. Preclinical data with the PI3K p110 gamma/delta inhibitor duvelisib also suggests its effectiveness in patients with C481S BTK mutations [9]. Data suggest that The Syk inhibitor entospletinib (GS-9973) can overcome PLCG2 mutations, and thus likely could also be beneficial in the presence of upstream mutations as well. Preliminary data showed a response rate of $28 \%$ among patients with a median exposure of 16 weeks (range 1-60) for ibrutinibresistant patients [9].

\section{Other promising drugs and targets}

Targeting of BTK with a reversible inhibitor that binds outside of C481 may be an ideal therapy. GDC-0853, ARQ531, and SNS-062 are all reversible BTK inhibitors that have shown preclinical efficacy. For those with PLCG2 mutations or BTK mutations, PKC $\beta$ might also be clinically relevant. Among potential others, this includes HSP90 inhibitors, HDAC inhibitors, and XPO1 inhibitors [9].

\section{References}

1. Lee CS, Rattu MA, Kim SS (2016) A review of a novel, Bruton's tyrosine kinase inhibitor, ibrutinib. J Oncol Pharm Practice 22(1): 92-104.

2. Marini BL, Samanas L, Perissinotti AJ (2016) Expanding the armamentarium for chronic lymphocytic leukemia: A review of novel agents in the management of chronic lymphocytic leukemia. J Oncol Pharm Practice pp: 1-16.

3. Dreyling M, Jurczak W, Jerkeman M, Silva RS, Rusconi C, Trneny M, et al. (2016) Ibrutinib versus temsirolimus in patients with relapsed or refractory mantle cell lymphoma: an international, randomised, openlabel, phase 3 study. Lancet 387: 770-778.

4. Mertens D, Stilgenbauer S (2017) Ibrutinib-resistant CLL: unwanted and unwonted!. Blood 129 (11): 1406-1409.

5. Zhang SQ, Smith SM, Zhang SY, Wang YL (2015) Mechanisms of ibrutinib resistance in chronic lymphocytic leukemia and non-Hodgkin lymphoma. Br J Haematol 170(4): 555-456.

6. Komarova NL, Burgerc JA, Wodarza D (2014) Evolution of ibrutinib resistance in chronic lymphocytic leukemia (CLL). PNAS 111(38): 13906-13911.

7. Winqvist M, Asklid A, Andersson PO, Karlsson K, Karlsson C, et al. (2016) Real -world results of ibrutinib in patients with relapsed or refractory chronic lymphocytic leukemia: data from 95 consecutive patients treated in a compassionate use program. A study from the Swedish Chronic Lymphocytic Leukemia Group. Haematologica 101(12): 1573-1580.

8. Woyach JA, Furman RR, Liu TM, Ozer HG, Zapatka M, et al. (2014) Resistance Mechanisms for the Bruton's tyrosine kinase inhibitor ibrutinib. N Engl J Med 370: 2286-2294.

9. Woyach JA (2017) How I manage ibrutinib-refractory chronic lymphocytic leukemia. Blood 129: 1270-1294.

10. Wallace N, Wong E, Cooper D, Chao H (2016) A case of new-onset cardiomyopathy and ventricular tachycardia in a patient receiving ibrutinib for relapsed mantle cell lymphoma. Clinical Case Reports 4(12): 1120-1121.

11. Jain P, Keating MI, Wierda W, Estrov Z, Ferrajoli A, et al. (2015) Outcomes of patients with chronic lymphocytic leukemia after discontinuing ibrutinib. Blood 125(13): 2062-2067.

12. Furman RR, Cheng S, Lu P, Setty M, Perez AR, et al. (2014) Ibrutinib resistance in chronic lymphocytic leukemia. N Engl J Med 370 (24): 2352-2354.

\section{Your next submission with Juniper Publishers} will reach you the below assets

Commons Attribution 4.0 License

DOI: $10.19080 /$ CTOIJ.2017.04.555640 